\section{NSCALE II: A program to analyze and score multiscale surveys and test batteries}

\author{
J. J. RAY and R. S. BOZEK \\ Department of Sociology, University of New South Wales, \\ Kensington 2033, Australia
}

Psychologists, sociologists, and educationists commonly administer to groups of subjects several additive scales at once, from cognitive test batteries to multiphasic personality inventories to Likert-type attitude scale questionnaires. When used for research purposes, each scale must be separately analyzed for adequacy and scored for each individual. Then, usually, the intercorrelations between the scales and their correlations with other variables are computed. NSCALE II is a program that carries out all these tasks in one run. It has sufficient flexibility to handle any mix of cognitive, personality, and Likert-type attitude scales. There is even an option to score and analyze one scale as both a Likert and a Thurstone scale. Cognitive tests can be scored either from raw data or from marked data. When "balanced" attitude or personality scales are used, the program also carries out all the reverse scoring required. There is also provision for scales to be analyzed both as separate subscales and as part of one larger overall scale. The total scores for each person on each scale can be put out both as a card deck suitable for input into further analyses and as a complete list on the line printer.

Input. All input is presently via cards. It is assumed that item responses have been coded by subject, that is, all answers of Subject 1 are on the first card (or set of cards), and so on. Parameter cards to specify job details are required between the program and data decks and details of these are given in a separate manual.

Output. The output gives the total scores for each individual on each scale. Then come item means, standard deviations, and item-to-total correlations (overlap corrected). The latter enable evaluation of the contribution of each item to what its scale measures.
Then come the coefficient-alpha reliability statistic and estimated mean interitem correlation for each scale considered as a whole. The former is the single most widely used index of scale adequacy. The means and standard deviations for all total scale scores and other (demographic) variables read in are then output. Next come the correlations between all scales and between the scales and demographic variables. Finally, the matrix of interscale correlations is cluster analyzed using McQuitty's (1961) method of elementary factor analysis.

Portability and Cost. The program has been used on a wide variety of machines but is presently running on a Cyber 70. It is therefore written in the CDC version of FORTRAN. Changes required to put it back into standard FORTRAN IV are minimal. The program comprises 440 lines of code and takes $117 \mathrm{~K}$ (octal) of core storage with present dimensions. Run-times vary between 5 and $10 \mathrm{sec}$ for most jobs. An entire research project can be analyzed at once for $\$ 1$ or $\$ 2$.

Restrictions and Capacity. The program is dimensioned for up to 220 scale items distributed among up to 23 scales of up to 50 items each. A much larger number of items could be handled with little extra call on core storage if the total number of scales into which they could fall were reduced.

Availability. Up-to-date listing and detailed program manual are available free of charge from the senior author at the Department of Sociology, University of New South Wales, P.O. Box 1, Kensington 2033, Australia. An earlier version of the program with lesser flexibility was reported in Ray (1972).

\section{REFERENCES}

McQuitTy, L. C. Elementary factor analysis. Psychological Reports, 1961, 9, 71-78.

RAY, J. J. NSCALE-A program to analyze and score a scale battery. Behavioral Science, 1972, 17, 490-491.

(Accepted for publication April 6, 1979.) 